\title{
IMMUNOLOGY
}

\section{Studying human lung infection in mice}

\author{
Mice implanted with human lung tissue model pathogen infection and \\ immune responses.
}

1 uman-mouse chimeric models (humanized mice), generated by engrafting human tissues and/ or stem cells into immunodeficient mice, are powerful tools for in vivo studies of human pathogens. However, for humanspecific pathogens that only infect nonhematopoietic cells, the lack of target human cell types in mouse models can limit their utility in basic and translational research. "We realized that there was a large need for preclinical in vivo models that could be used to study these human pathogens, novel treatment and prevention approaches, as well as vaccine vectors and the human immune response that they elicit," says Angela Wahl of the University of North Carolina School of Medicine.

Lung tissue harbors various cell types that are involved in the infection and immunity of many human pathogens, and it could be an ideal source for humanizing mouse models. Pursuing this line, Wahl, J. Victor Garcia and colleagues created lungonly mice (LoM) by implanting human lung tissue subcutaneously into the backs of immunodeficient mice. While ectopic and not ventilated, these lung implants develop characteristic structures of the human lung with extensive vasculature. A number of challenging pathogens, including Middle East respiratory syndrome coronavirus, Zika virus, mycobacteria, respiratory syncytial virus and cytomegalovirus, were found to replicate in the lung implants after inoculation. Moreover, the model can recapitulate the effect of pre-exposure prophylaxis of ganciclovir, an antiviral agent, on cytomegalovirus (HCMV) replication.

To further model human immunity against pathogens in vivo, the team created a second model, BLT-L mice, by implanting autologous bone marrow-liver-thymus tissue and lung tissue into the immunodeficient mice. Human innate and adaptive immune cells are systematically reconstituted in BLT-L mice, thus facilitating examining various immune responses upon infection. As an example, while HCMV causes acute infection in BLT-L mice, sustained subsequent humoral and $\mathrm{T}$ cell responses are ultimately capable of containing the replication to background levels, reminiscent of latent infection in healthy individuals.

"We were surprised at just how many different human pathogens replicated in the models and at how well the human immune cells in BLT-L mice could control infection, just like they would in people," says Wahl.

To further mimic chronic HCMV infection that might occur in immunesuppressed individuals, such as transplant and AIDS patients, BLT-L mice were challenged by multiple exposures to HCMV. Antibody induction and class-switching were elicited, as well as HCMV-specific $\mathrm{CD} 4^{+}$and $\mathrm{CD}^{+} \mathrm{T}$ cell responses. These observations also suggest the BLT-L mice may be useful for vaccine testing in the future.

These models hold potential for investigating the pathogenicity of some of most dangerous and stubborn microbes. "We envisioned that LoM and BLT-L mice could be used to study important emerging human pathogens like severe acute respiratory syndrome coronavirus (SARS), Middle East respiratory syndrome coronavirus (MERS), and tuberculosis. These pathogens are BSL-3 pathogens requiring additional safety precautions. Therefore, we had to carefully consider the location of the human lung implants in the models so that they could be easily and safely manipulated in a BSL-3 facility," says Wahl. Regarding their translational value, she and colleagues see profound applications in the development, evaluation and implementation of novel approaches to treatment and prevention. "Our plan is to continue to demonstrate the outstanding applicability of these models in multiple areas of basic and translational research and the type of fundamental insight that they can provide. We believe that this will encourage others to take advantage of these tools for discovery."

\section{Lin Tang}

Published online: 28 November 2019 https://doi.org/10.1038/s41592-019-0672-8

Research paper

Wahl, A. et al. Precision mouse models with expanded tropism for human pathogens. Nat. Biotechnol. 37, 1163-1173 (2019). nature masterclasses

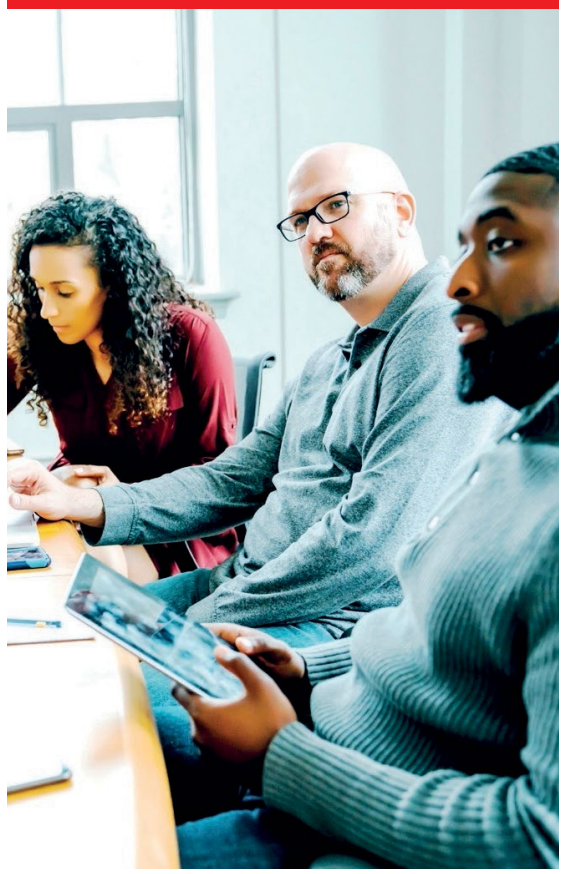

Workshops in

Scientific Writing and Publishing

Helping researchers turn great science into great papers.

Delivered by Nature Research

journal editors and hosted at institutions worldwide.

\section{Find out more at \\ masterclasses.nature.com}

W masterclasses.nature.com

in Follow us on Linkedln 